\title{
Flujo vaginal y semen: La microbiota de las relaciones sexuales
}

\section{Vaginal Flow and Semen: The Microbiote of Sexual Relations}

\author{
Valentina Restrepo Arenas ${ }^{1}$ Valentina Velásquez Rivera ${ }^{1}{ }^{\circledR}$ Jenniffer Puerta Suárez ${ }^{1}{ }^{\circledR}$ \\ Walter D. Cardona Maya10 \\ ${ }^{1}$ Departamento de Microbiología y Parasitología, Grupo
Reproducción, Facultad de Medicina, Universidad de Antioquia,
Antioquia, Colombia

\begin{abstract}
Address for correspondence Walter D. Cardona Maya, Bact, MSc, PhD, Departamento de Microbiología y Parasitología, Grupo Reproducción, Facultad de Medicina, Universidad de Antioquia, Antioquia, Colombia (e-mail: wdario.cardona@udea.edu.co).
\end{abstract}

Urol Colomb 2021;30:140-144.

\section{Resumen \\ Palabras claves \\ - Microbiota \\ - Fertilidad \\ - Relaciones sexuales \\ - Flujo vaginal \\ - Semen \\ - Bacterias}

Durante las relaciones sexuales, se da el intercambio de especies bacterianas entre las parejas, siendo las relaciones sexuales un factor crucial en la modificación de la microbiota genital. En el presente artículo de reflexión, se analizó el efecto de las relaciones sexuales sobre el intercambio de la microbiota vaginal y seminal. Las parejas que sostienen relaciones sexuales sin protección comparten especies bacterianas que podrían influir negativamente o positivamente sobre los parámetros seminales, como los Lactobacillus con potencial probiótico para el mantenimiento de la calidad del semen.

During sexual intercourse there is an exchange of bacterial species between couples, a crucial factor in modifying the genital microbiota. In this reflection article, the effect of sexual intercourse on the exchange of vaginal and seminal microbiota was analyzed. Couples who have unprotected sexual intercourse share bacterial species could affect positive or negatively the seminal parameters, such as Lactobacillus with probiotic potential for maintaining semen quality.

- vaginal discharge

- semen

- bacteria

\section{Introducción}

Uno de los eventos más importantes para la vida del neonato es el cambio del ambiente uterino aparentemente estéril a su colonización por microorganismos al nacer, sin embargo, pocos microorganismos logran colonizar permanentemente algunos sitios corporales probablemente por los mecanismos de adaptación de algunas bacterias que no soportan los ambientes corporales como el $\mathrm{pH}$, la cantidad de oxígeno y las estructuras epiteliales. ${ }^{1,2}$ Esa colonización es de gran importancia en la protección de todos los órganos, particularmente de la piel, para que no se establezcan microorganismos patógenos, actuando como un sistema de defensa por ocupación con bacterias benéficas. Esas bacterias desarrollan proteínas de superficie (adhesinas), que les permite promover la unión a los tejidos del huésped y a los received

July 22, 2020

accepted

September 15, 2020

published online

February 4, 2021
DOI https://doi.org/

$10.1055 / \mathrm{s}-0040-1721325$.

ISSN 0120-789X.

e ISSN 2027-0119. (c) 2021. Sociedad Colombiana de Urología. All rights reserved. This is an open access article published by Thieme under the terms of the Creative Commons Attribution-NonDerivative-NonCommercial-License, permitting copying and reproduction so long as the original work is given appropriate credit. Contents may not be used for commercial purposes, or adapted, remixed, transformed or built upon. (https://creativecommons.org/ licenses/by-nc-nd/4.0/)

Thieme Revinter Publicações Ltda., Rua do Matoso 170, Rio de Janeiro, RJ, CEP 20270-135, Brazil 
biomateriales recubiertos de proteínas, un paso esencial durante el proceso de colonización del huésped. ${ }^{3,4}$

En los últimos años se ha determinado que la piel humana, los intestinos, la cavidad oral, la vagina y la uretra, alojan comunidades microbianas asociadas con la salud humana, incluyendo la salud sexual y reproductiva. ${ }^{5-8}$ La presencia preconcepcional de la microbiota en los órganos reproductivos femeninos y masculinos sugiere que el proceso de interacción entre gametos se lleva a cabo en un ambiente que no es estéril lo que contribuye, de alguna manera, al éxito reproductivo. ${ }^{9}$

La microbiota de los tractos urogenitales masculino y femenino es dinámica y en condiciones normales proporciona defensa antimicrobiana contra la infección por microorganismos patógenos, ${ }^{10}$ ayudando a reducir su potencial de infiltración; ${ }^{9}$ sin embargo, debido al uso generalizado de antibióticos, al estrés y otros factores ambientales, la relación huésped-microbiota pasa de ser beneficiosa, simbiótica y bien equilibrada, a una disbiosis, la cual se refleja por la interrupción en el equilibrio local o la composición de la microbiota, asociado con inflamación y enfermedad. ${ }^{2,9}$

Hasta la fecha, la información sobre el papel de las bacterias en el semen es controversial, pero es interesante observar que muchas bacterias presentes en el semen están estrechamente relacionadas con las que se encuentran en la vagina, además que durante las relaciones sexuales suceden cambios significativos en la microbiota genital. ${ }^{11}$ Por lo tanto, el objetivo del presente artículo de reflexión, es indagar en las publicaciones sobre la microbiota seminal y vaginal donde se mencione las relaciones sexuales como factor alterante, determinando así las especies bacterianas comúnmente encontradas, el papel de las relaciones sexuales en la modificación de la microbiota y donde ella a su vez, tenga posibles implicaciones en la calidad seminal.

\section{Métodos}

Con el propósito de determinar la relación entre las bacterias y las relaciones sexuales, se buscó información publicada sobre los temas relacionados con microbiota vaginal, microbiota seminal y las relaciones sexuales asociada al cambio de microbiota, mediante la búsqueda de material bibliográfico a través de PubMed y ScienceDirect (junio 3 de 2020) sin límite de tiempo, empleando los términos "microbiota vaginal, microbiota seminal, sexual intercourse and semen, sexual intercourse and microbiota, seminal quality and sexual intercourse" obteniendo un total de 6,509 artículos en ambas bases de datos. Se preseleccionaron artículos originales, revisiones del tema, investigaciones experimentales $\mathrm{y}$ capítulos de libros, reduciendo el número de artículos a 115 que cumplieron con los criterios de inclusión. Por último se indagó cuál de esa bibliografía aportaba al objetivo de la presente revisión narrativa para finalmente seleccionar un total de 32 artículos que presentaban la información más relevante según el criterio de los autores, y de forma adicional se revisó la bibliografía reportada en cada artículo con el propósito de ampliar los resultados.

\section{La microbiota vaginal}

La microbiota cumple un papel fundamental en el mantenimiento de la salud humana y se ubica en diferentes sitios corporales colonizándolos y adaptándose para sobrevivir, incluso en el tracto genital se encuentran especies bacterianas implicadas en la salud sexual y el éxito reproductivo. ${ }^{1-7}$ Específicamente, en el tracto reproductivo femenino (vagina, vulva y el cuello uterino), hay una colonización por microorganismos y se ha observado que la microbiota vaginal está compuesta por una comunidad moderadamente diversa. ${ }^{6,9,12}$

La evidencia muestra que las mujeres en edad fértil poseen una microbiota vaginal donde predominan una o dos especies de Lactobacillus (L.crispatus, L.jensenii, L. gasseri y L. Iners), que protegen el ecosistema vaginal mediante la producción de moléculas con efecto antimicrobiano directo como el ácido láctico y el peróxido de hidrógeno, además de preservar el pH ácido del microambiente vaginal, ${ }^{13-16}$ son metabolitos con efecto antiviral directo que no solo modulan la respuesta inmune frente a los patógenos sino también mantienen la calidad de vida de las mujeres. ${ }^{17-22}$

Los cambios en esa microbiota genital están influenciados por procesos fisiológicos como la edad, la concentración hormonal y el embarazo. ${ }^{23-25}$ Además, varias publicaciones postulan que las relaciones sexuales son también un factor crucial en la modificación de la microbiota genital demostrando que al evaluar la microbiota seminal de hombres que sostenían relaciones sexuales con su pareja, se identificaron bacterias compartidas con la microbiota vaginal después del coito, incluso esos individuos tuvieron una microbiota más diversa. ${ }^{6,16-20}$

La microbiota vaginal es un ecosistema abierto que puede verse significativamente afectado tanto por factores patológicos como fisiológicos, y dentro de esos últimos, se encuentran la variación en las concentraciones hormonales, las etapas de vida de la mujer, el ciclo reproductivo y el embarazo. ${ }^{1,23,24}$ Igualmente, la ingesta de antibióticos, uso de duchas vaginales, tampones, alimentación, el tabaco y las relaciones sexuales estarán implicadas en los cambios de la microbiota. $^{8,25-28}$

\section{Microbiota seminal}

De otro lado, la caracterización de la microbiota del tracto genital masculino siempre ha sido rezagada con respecto a las investigaciones en otros sitios del cuerpo, ${ }^{29}$ poco o nada se conoce en ese campo, y aún está por definir cómo cambia ese ambiente a lo largo de la vida y que hitos son importantes generadores de alteraciones. En comparación con la microbiota vaginal, el semen posee al parecer una microbiota más, se reporta presencia de especies de Lactobacillus spp., Staphylococcus, Streptococos, bacterias corineformes y varios anaerobios. ${ }^{4,15,30,31}$

La microbiota del tracto genital masculino es principalmente aquella que se encuentra en la uretra y en el surco coronal, ${ }^{32,33}$ sin embargo, cada glándula y porción 
anatómica del tracto genitourinario puede contribuir con la presencia de microorganismos. Se ha reportado la presencia de cocos gran positivos, corniformes y algunos anaerobios. ${ }^{30}$

El semen posee una mayor diversidad de bacterias en comparación con la microbiota vaginal y se ha evidenciado que provoca una respuesta inmune adaptativa en los tejidos cervicales, ${ }^{9,23}$ que induce un cambio significativo en la microbiota vaginal después del coito sin protección y que es un medio para la transmisión de microorganismos, contribuyendo al desarrollo de infecciones de transmisión sexual (ITS), ${ }^{8}$ e incluso posibles cambios en la microbiota, sin embargo existe poca información sobre la transmisión de especies bacterianas e interacciones beneficiosas entre la microbiota de parejas (-Fig. 1).

\section{Microbiota y las relaciones sexuales}

Compartir la microbiota durante las relaciones sexuales podría posibilitar los procesos infecciosos e inflamatorios los cuales comprometerían la calidad y concentración de espermatozoides, parámetros que podrían estar asociados con efectos negativos de la fertilidad. 1,22,29,30,32,33 Sin embargo, la información además de ser poca es controversial, pues otros estudios mencionan el papel necesario de las bacterias en el semen, particularmente es el caso de Lactobacillus spp. que mantienen la calidad seminal, contrarrestan la influencia negativa de otros patógenos y podrían ser factor protector para el desarrollo de otras alteraciones genitourinarias, ${ }^{4,17,34,35}$ hallazgos que se ajustan a la hipótesis de que la disbacteriosis en el tracto genital masculino podría estar relacionado con la prostatitis crónica. ${ }^{36}$

En el presente artículo de reflexión se encontraron publicaciones que establecen el papel cooperativo de la microbiota en el mantenimiento de la salud humana. ${ }^{3-10}$ En la vagina, predominan las especies de Lactobacillus spp., mientras que el semen posee una mayor diversidad bacteriana y alberga perfiles únicos de microbiota que parecen conservarse en las poblaciones humanas. ${ }^{6,12-22,29,30,32,33,37,38}$

\section{Factores asociados al cambio de la microbiota}

Al igual que la edad, los cambios hormonales, la alimentación, el embarazo y el tabaco, ${ }^{1,23-25}$ las relaciones sexuales están implicadas en la modificación de la microbiota genital, en hombres sin experiencia sexual la concentración bacteriana total es más baja y poseen una diversidad bacteriana menor respecto a los hombres sexualmente experimentados. ${ }^{30}$ Se han identificado varios taxones bacterianos en el semen que se asocian con las comunidades vaginales, ${ }^{39}$ lo que sugiere que las parejas sexualmente activas pueden compartir especies bacterianas como los Lactobacillus spp. ${ }^{8,40}$ Además, la composición de la microbiota genital es similar entre compañeros sexuales, con mayor diversidad y riqueza de microorganismos que las personas no convivientes. ${ }^{28}$

Aunque algunos mecanismos no están claramente dilucidados o incluso pueden ser divergentes entre los diferentes estudios, un evento probable durante ese intercambio de microbiota, es que la relación microorganismo-humano puede tornarse de mutualista a parasitaria, llevando consigo procesos infecciosos e inflamatorios los cuales están relacionados con la infertilidad. ${ }^{41}$

\section{Microbiota y fertilidad}

La infertilidad es una condición cada vez más común, ${ }^{42}$ la bacteriospermia y el reclutamiento de leucocitos seminales, el deterioro de la espermatogénesis, la función de los espermatozoides y la obstrucción del tracto genital, pueden afectar la calidad de los espermatozoides y por ende la fertilidad masculina. ${ }^{43,44}$ La presencia bacteriana en el semen reduce la concentración y la viabilidad de los espermatozoides, ${ }^{45}$ es asi como la $E$. coli uropatógena aislada del semen induce la activación de leucocitos polimorfonucleares con la liberación

\section{Tracto reproductivo femenino}

\section{Tracto reproductivo masculino}

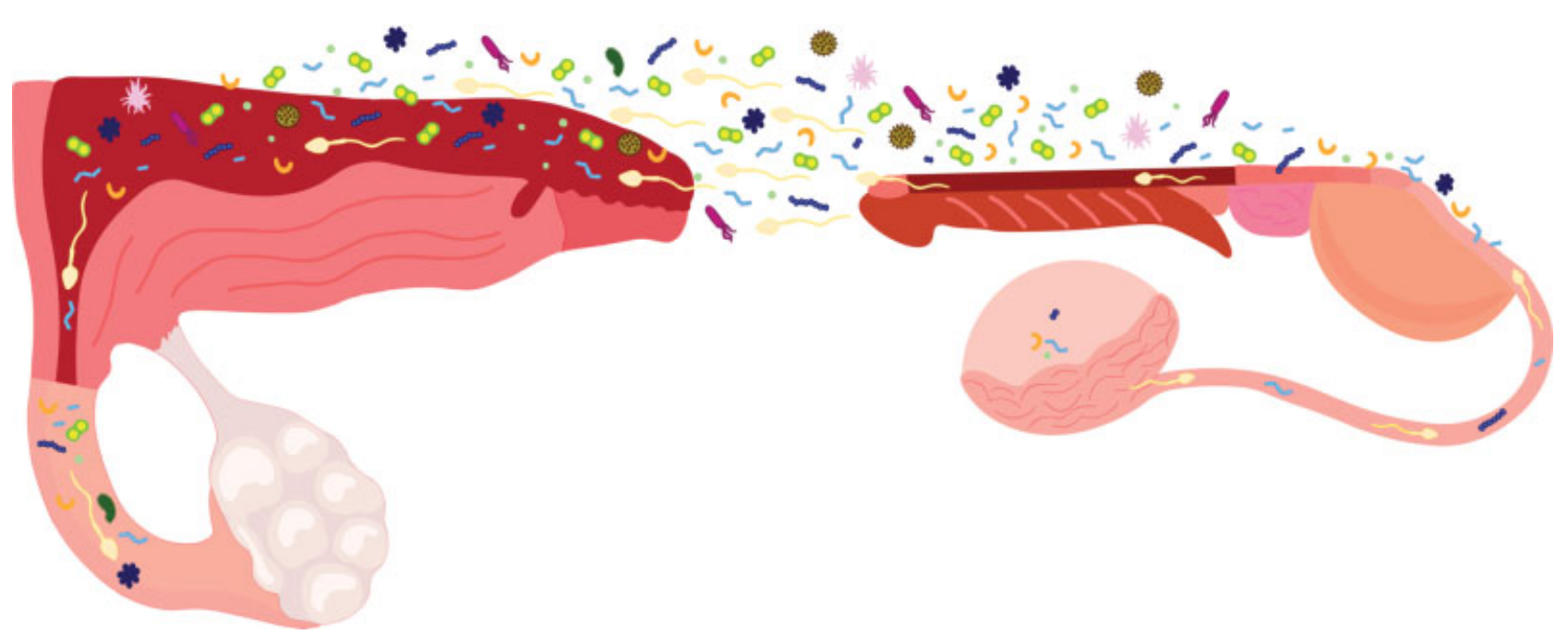

Fig. 1 Intercambio de microbiota durante las relaciones sexuales. Especies de microorganismos se intercambian entre el tracto reproductivo femenino y el masculino durante las relaciones sexuales. 
de citocinas y especies reactivas de oxígeno, siendo, ese último, dañino por inducir la peroxidación lipídica y la capacitación temprana de espermatozoides, ${ }^{46}$ así como la movilidad, la apoptosis, la necrosis e incluso el índice de fertilidad. ${ }^{47} \mathrm{Sin}$ embargo, la mayoría de los datos sobre las interacciones entre los espermatozoides y las bacterias se derivan de estudios in vitro, en condiciones que pueden no imitar con precisión las condiciones in vivo. ${ }^{10,35}$

Aunque siempre se asoció la bacteriospermia negativamente con la fertilidad y la calidad seminal, algunos estudios recientes indican no ser perjudicial, al contrario, parece ser necesaria para el buen funcionamiento de los espermatozoides, ${ }^{24}$ es así como la presencia de Lactobacillus como grupo predominante en el semen de individuos con parámetros seminales normales podrían no solo ser un probiótico potencial para el mantenimiento de la calidad del semen, sino que también podría ser útil para contrarrestar la influencia negativa de bacterias del género Prevotella spp. $y$ Pseudomonas spp. ${ }^{5}$

En conclusión, como se observa a lo largo de esta revisión, las relaciones sexuales modifican la microbiota genital mediante un proceso que se considera dinámico, el cual inicia una vez ingresa el semen en el tracto genital femenino, e incluso, los espermatozoides servirían como el vehículo para algunas especies bacterianas, además, durante la relación sexual, el hombre tomará parte de la microbiota de la cavidad vaginal de la pareja. Basados en eso, cuando las parejas tienen relaciones sexuales sin protección, la microbiota se modifica y por ende comienzan a compartir especies bacterianas.

Por lo tanto, cada día es más importante la microbiota de las relaciones sexuales, que aparece como resultado del intercambio entre la microbiota vaginal y seminal de las parejas tras el coito y que estará involucrada en el éxito reproductivo, ( - Fig. 1) donde especies bacterianas presentes en el flujo vaginal favorecerán a los espermatozoides e incluso contrarrestarán efectos negativos de otras especies bacterianas presentes dúrate el coito. Finalmente, se requieren más investigaciones al respecto para establecer con claridad cuál es el papel de esas especies y cómo podrían afectar negativamente, o quizás positivamente, los parámetros seminales, pues la información disponible hasta la fecha es controversial.

\section{Conflicto de intereses}

Los autores declaran no tener ningún conflicto de intereses.

\section{Referencias}

1 Wilson M. Bacteriology of humans: an ecological perspective. John Wiley \& Sons; 2009

2 Tungland B. Human Microbiota in Health and Disease: From Pathogenesis to Therapy. Academic press; 2018

3 Viela F, Mathelié-Guinlet M, Viljoen A, Dufrêne YF. What makes bacterial pathogens so sticky? Mol Microbiol 2020;113(04):683-690

4 Larsen B, Monif GR. Understanding the bacterial flora of the female genital tract. Clin Infect Dis 2001;32(04):e69-e77

5 Weng SL, Chiu CM, Lin FM, et al. Bacterial communities in semen from men of infertile couples: metagenomic sequencing reveals relationships of seminal microbiota to semen quality. PLoS One 2014;9(10):e110152

6 Robertson SA, Sharkey DJ. Seminal fluid and fertility in women. Fertil Steril 2016;106(03):511-519

7 Ravel J, Gajer P, Abdo Z, et al. Vaginal microbiome of reproductiveage women. Proc Natl Acad Sci U S A 2011;108(Suppl 1):4680-4687

8 Mändar R, Punab M, Borovkova N, et al. Complementary seminovaginal microbiome in couples. Res Microbiol 2015;166 (05):440-447

9 Schoenmakers S, Steegers-Theunissen R, Faas M. The matter of the reproductive microbiome. Obstet Med 2019;12(03):107-115

10 Berktas M, Aydin S, Yilmaz Y, Cecen K, Bozkurt H. Sperm motility changes after coincubation with various uropathogenic microorganisms: an in vitro experimental study. Int Urol Nephrol 2008;40(02):383-389

11 Gallo MF, Warner L, King CC, et al. Association between semen exposure and incident bacterial vaginosis. Infect Dis Obstet Gynecol 2011;2011:842652

$12 \mathrm{Nam} \mathrm{H}$, Whang K, Lee Y. Analysis of vaginal lactic acid producing bacteria in healthy women. J Microbiol 2007;45(06):515-520

13 Lamont RF, Sobel JD, Akins RA, et al. The vaginal microbiome: new information about genital tract flora using molecular based techniques. BJOG 2011;118(05):533-549

14 Borovkova N, Korrovits P, Ausmees K, et al. Influence of sexual intercourse on genital tract microbiota in infertile couples. Anaerobe 2011;17(06):414-418

15 Linhares IM, Summers PR, Larsen B, Giraldo PC, Witkin SS. Contemporary perspectives on vaginal $\mathrm{pH}$ and lactobacilli. Am J Obstet Gynecol 2011;204(02):120.e1-120.e5

16 Boskey ER, Telsch KM, Whaley KJ, Moench TR, Cone RA. Acid production by vaginal flora in vitro is consistent with the rate and extent of vaginal acidification. Infect Immun 1999;67(10):5170-5175

17 Anahtar MN, Byrne EH, Doherty KE, et al. Cervicovaginal bacteria are a major modulator of host inflammatory responses in the female genital tract. Immunity 2015;42(05):965-976

18 Conti C, Malacrino C, Mastromarino P. Inhibition of herpes simplex virus type 2 by vaginal lactobacilli. J Physiol Pharmacol 2009;60(Suppl 6):19-26

19 Chan RC, Reid G, Irvin RT, Bruce AW, Costerton JW. Exclusión competitiva de uropatógenos de células uroepiteliales humanas por células enteras de Lactobacillus y fragmentos de pared celular. Infección e inmunidad 1985;47(01):84-89

20 Ranjeva S, Baskerville EB, Dukic V, et al. Recurring infection with ecologically distinct human papillomavirus (HPV) types explains high prevalence and diversity. bioRxiv 2017;•••:179341

21 Schwiertz A. Microbiota of the human body. Adv Exp Med Biol 2016;902: $\mathrm{V}$

22 Ellis AK, Verma S. Quality of life in women with urinary tract infections: is benign disease a misnomer? J Am Board Fam Pract 2000;13(06):392-397

23 Ma B, Forney LJRavel. J. Microbioma vaginal: repensar la salud y la enfermedad. Revisión anual de microbiología 2012

24 Witkin SS. El microbioma vaginal, los mecanismos de defensa antimicrobianos vaginales y el desafío clínico de reducir el parto prematuro relacionado con la infección. BJOG: An International Journal of Obstetrics \& Gynecology 2015

25 Agüero SDLÁM. Microbiota y disbiosis vaginal. Revista Medica Sinergia 2019;4(01):3-13

26 Romero R, Hassan SS, Gajer P, et al. The vaginal microbiota of pregnant women who subsequently have spontaneous preterm labor and delivery and those with a normal delivery at term. Microbiome 2014;2(01):18

27 Mändar R. Microbiota of male genital tract: impact on the health of man and his partner. Pharmacol Res 2013;69(01):32-41

28 Dill-McFarland KA, Tang ZZ, Kemis JH, et al. Close social relationships correlate with human gut microbiota composition. Sci Rep 2019;9(01):703 
29 Nelson DE, Dong Q Van der Pol B, et al. Bacterial communities of the coronal sulcus and distal urethra of adolescent males. PLoS One 2012;7(05):e36298

30 Ramírez-Arango J, Ruiz LA, Maldonado GA, Herrera MC. Micturating cystourethrography: a review of the diagnostic method and its usefulness in the study of common disorders. Anales de Radiología, México 2017;16(01):20-30

31 Reid G, Bruce AW, McGroarty JA, Cheng KJ, Costerton JW. Is there a role for lactobacilli in prevention of urogenital and intestinal infections? Clin Microbiol Rev 1990;3(04):335-344

32 Kiessling AA, Desmarais BM, Yin HZ, Loverde J, Eyre RC. Detection and identification of bacterial DNA in semen. Fertil Steril 2008;90 (05):1744-1756

33 Mändar R, Türk S, Korrovits P, Ausmees K, Punab M. Impact of sexual debut on culturable human seminal microbiota. Andrology 2018;6(03):510-512

34 Baud D, Pattaroni C, Vulliemoz N, Castella V, Marsland BJ, Stojanov M. Sperm microbiota and its impact on semen parameters. Front Microbiol 2019;10:234

35 Mändar R, Punab M, Korrovits P, et al. Seminal microbiome in men with and without prostatitis. Int J Urol 2017;24(03):211-216

36 Liu L, Yang J, Lu F. Urethral dysbacteriosis as an underlying, primary cause of chronic prostatitis: potential implications for probiotic therapy. Med Hypotheses 2009;73(05):741-743

37 Bayigga L, Kateete DP, Anderson DJ, Sekikubo M, Nakanjako D. Diversity of vaginal microbiota in sub-Saharan Africa and its effects on HIV transmission and prevention. Am J Obstet Gynecol 2019;220(02):155-166

38 Atassi F, Brassart D, Grob P, Graf F, Servin AL. Vaginal Lactobacillus isolates inhibit uropathogenic Escherichia coli. FEMS Microbiol Lett 2006;257(01):132-138

39 Hou D, Zhou X, Zhong X, et al. Microbiota of the seminal fluid from healthy and infertile men. Fertil Steril 2013;100(05):1261-1269
40 Dohle GR, Colpi GM, Hargreave TB, Papp GK, Jungwirth A. Weidner, WEAU y EAU Grupo de trabajo sobre infertilidad masculina. Pautas de la EAU sobre infertilidad masculina. Urología europea 2005;48(05):703-711

41 Puerta-Suárez J, Giraldo M, Cadavid ÁP, Cardona-Maya W. Infecciones bacterianas del tracto reproductivo masculino y su papel en la fertilidad. Rev Chil Obstet Ginecol 2014;79(03): 209-217

42 Brugo-Olmedo S, Chillik C, Kopelman S. Definición y causas de la infertilidad. Rev Colomb Obstet Ginecol 2003;54(04):227-248

43 Pellati D, Mylonakis I, Bertoloni G, et al. Genital tract infections and infertility. Eur J Obstet Gynecol Reprod Biol 2008;140(01): 3-11

44 Domes T, Lo KC, Grober ED, Mullen JBM, Mazzulli T, Jarvi K. La incidencia y el efecto de bacteriospermia y leucocitos seminales elevados en los parámetros del semen. Fertilidad y esterilidad 2012;97(05):1050-1055

45 Enwurua CA, Iwalokuna B, Enwuru VN, Ezechi O, Oluwadun A. The effect of presence of facultative bacteria species on semen and sperm quality of men seeking fertility care. Afr J Urol 2016;22 (03):213-222

46 Villegas JV, Boguen R, Uribe P. Effect of Uropathogenic Escherichia coli on Human Sperm Function and Male Fertility. Escherichia coli-Recent Advances on Physiology, Pathogenesis and Biotechnological Applications. Amidou Samie, IntechOpen 2017. Doi: 10.5772/intechopen.68312 Available from: https://www. intechopen.com/books/-i-escherichia-coli-i-recent-advanceson-physiology-pathogenesis-andbiotechnological-applications/effect-of-uropathogenic-iescherichia-coli-i-on-human-sperm-function-and-male-fertility

47 Moretti E, Capitani S, Figura N, et al. The presence of bacteria species in semen and sperm quality. J Assist Reprod Genet 2009; 26(01):47-56 